\title{
Age plays an important role in the decision of definitive concurrent chemoradiotherapy (CCRT) for esophageal squamous cell carcinoma (ESCC): a propensity-score matched analysis of multicenter data (3JECROG R-02A)
}

\author{
Lan Wang ${ }^{1}$, Xin Wang ${ }^{2}$, Xuejiao Ren ${ }^{1}$, Chun Han $^{1}$, Zefen Xiao ${ }^{2}$, Shuchai Zhu ${ }^{1}$, Xueying Qiao ${ }^{1}$, \\ Zhiguo Zhou ${ }^{1}$, Wenbin Shen ${ }^{1}$, Junqiang Chen ${ }^{3}$, Qingsong Pang ${ }^{4}$, Wencheng Zhang ${ }^{4}$, Yidian Zhao ${ }^{5}$, \\ Xiaomin Wang ${ }^{5}$, Xinchen Sun ${ }^{6}$, Xiaolin Ge ${ }^{6}$, Kaixian Zhang ${ }^{7}$, Miaomiao Hu${ }^{7}$, Gaofeng Li $^{8}$, Miaoling Liu ${ }^{9}$, \\ Yadi Wang ${ }^{10}$
}

${ }^{1}$ Department of Radiation Oncology, The Fourth Hospital of Hebei Medical University, Shijiazhuang, China; ${ }^{2}$ Department of Radiation Oncology, National Cancer Center/National Clinical Research Center for Cancer/Cancer Hospital, Chinese Academy of Medical Sciences and Peking Union Medical College, Beijing, China; ${ }^{3}$ Department of Radiation Oncology, Teaching Hospital of Fujian Medical University, Fujian Provincial Cancer Hospital, Fuzhou, China; ${ }^{4}$ Department of Radiation Oncology, Tianjin Medical University Cancer Institute and Hospital/National Clinical Research Center for Cancer, Tianjin, China; ${ }^{5}$ Department 4th of Radiation Oncology, Anyang Cancer Hospital, Anyang, China; ${ }^{6}$ Department of Radiation Oncology, The First Affiliated Hospital of Nanjing Medical University, Nanjing, China; ${ }^{7}$ Department of Oncology, Tengzhou Central People's Hospital, Tengzhou, China; ${ }^{8}$ Department of Radiation Oncology, Beijing Hospital, Beijing, China; ${ }^{9}$ Department of Radiation Oncology, Affiliated Hospital of Hebei University, Baoding, China; ${ }^{10}$ Department of Radiation Oncology, the PLA Army General Hospital, Beijing, China

Contributions: (I) Conception and design: L Wang, C Han, Z Xiao; (II) Administrative support: C Han, Z Xiao; (III) Provision of study materials or patients: L Wang, X Wang, S Zhu, X Qiao, Z Zhou, W Shen, J Chen, Q Pang, W Zhang, Y Zhao, X Wang, X Sun, X Ge, K Zhang, M Hu, G Li, M Liu, Y Wang; (IV) Collection and assembly of data: L Wang, X Wang; (V) Data analysis and interpretation: L Wang, X Ren; (VI) Manuscript writing: All authors; (VII) Final approval of manuscript: All authors.

Correspondence to: Prof. Chun Han. Department of Radiation Oncology, The Fourth Hospital of Hebei Medical University, Shijiazhuang 050011, China. Email: hanchun2006@126.com; Prof. Zefen Xiao. Department of Radiation Oncology, National Cancer Center/National Clinical Research Center for Cancer/Cancer Hospital, Chinese Academy of Medical Sciences and Peking Union Medical College, Beijing 100021, China. Email: xiaozefen@sina.com.

Background: To examine the survival benefit of definitive concurrent chemoradiotherapy (CCRT) compared to radiotherapy alone in patients with esophageal squamous cell carcinoma (ESCC) using a real-world patient population.

Methods: This retrospective study included 2,762 patients with ESCC across ten medical centers in China from 2001 to 2017. A total of 1,133 patients received radiotherapy alone and 815 patients were treated with CCRT. The patient survival rates were assessed by propensity-score matching (PSM) and subgroup analysis.

Results: The baseline characteristics were significantly different between the two groups, with the CCRT group showing a higher proportion of males, younger patients, cervical/upper thoracic cancers, and worse $\mathrm{T}$ and $\mathrm{N}$ stages. There were no significant differences in the clinical characteristics between the two groups after PSM. Before PSM, the median overall survival (OS) rates were 31.2 and 24.1 months in the CCRT and RT alone groups, respectively, demonstrating the superior therapeutic effects (TEs) of the CCRT. However, the median OS rates were not significantly different between the two groups after PSM (32.6 and 39.4 months in the CCRT and radiotherapy alone groups, respectively). The subgroup analyses revealed that the median OS was significantly better in the CCRT group compared to the radiotherapy alone group $(37.5$ vs. 25.1 months, respectively) in patients less than 70 years of age [hazard ratio (HR) 0.782 , 95\% confidence interval (CI): 0.657 to 0.932 ]. In contrast, in patients 70 years of age and older, the 5 -year survival rate was poorer in the CCRT group (34.8\%) compared to the radiotherapy alone group (73.4\%). Therefore, CCRT was an independent poor prognostic risk factor (HR 3.206, 95\% CI: 2.168 to 4.740). 
Conclusions: CCRT may not be suitable for all patients with localized ESCC. Younger patients less than 70 years of age might benefit significantly from CCRT. However, in patients aged 70 years and older, the potential survival benefit of CCRT and the optimal combination treatment regimens require further investigation.

Keywords: Esophageal neoplasm; esophageal squamous cell carcinoma (ESCC); chemoradiotherapy; radiotherapy; prognosis

Submitted Feb 07, 2021. Accepted for publication May 21, 2021.

doi: $10.21037 /$ tcr-21-505

View this article at: https://dx.doi.org/10.21037/tcr-21-505

\section{Introduction}

Definitive concurrent chemoradiotherapy (CCRT) is a standard therapeutic modality in patients with inoperable esophageal cancer, based on the evidence derived primarily from two trials (RTOG8501 (1-3) and RTOG9405 (4). However, in the real clinical setting, a considerable number of patients have difficulties tolerating CCRT because of their older age and physical weakness, combined with a wide range of lesions, large tumor burden, and comorbidities. In addition, high-level evidence confirming or replicating the results of the RTOG8501 and RTOG9405 trials have been lacking in China, where squamous cell carcinoma is the main pathological type of esophageal cancer. Furthermore, numerous studies (5-10) have shown that the efficacy of radiotherapy alone is much better than that reported in the RTOG8501 trial (5-year survival rate of $0 \%$ ). Therefore, a key problem is to find the truly applicable population for definitive CCRT and avoid potential high-risk treatment, this is also the purpose and innovation of this research. Based on this, we retrospectively analyzed 2,762 patients with esophageal squamous cell carcinoma (ESCC) treated by definitive 3-dimensional conformal radiotherapy (3DCRT) or intensity modulated radiotherapy (IMRT) in ten medical centres in China to elucidate the subgroups who might benefit from CCRT compared with radiotherapy alone. We present the following article in accordance with the STROBE reporting checklist (available at https://dx.doi. org/10.21037/tcr-21-505).

\section{Methods}

\section{Data source}

The present study included data obtained from the medical records of ten medical centers (The Fourth
Hospital of Hebei Medical University, National Cancer Center/National Clinical Research Center for Cancer/ Cancer Hospital, Fujian Cancer Hospital/Fujian Medical University Cancer Hospital, Tianjin Medical University Cancer Institute and Hospital/National Clinical Research Center for Cancer, Anyang Cancer Hospital, First Affiliated Hospital of Nanjing Medical University, Tengzhou Central People's Hospital, Beijing Hospital, National Center of Gerontology, Affiliated Hospital of Hebei University, and PLA Army General Hospital). The clinical cases included in each center were strictly controlled by experienced researchers, it was requested that the medical records provided access to high-quality and sufficient information regarding patient demographics, primary tumor characteristics, pathology, radiation modality and dose, treatment regimens, toxicity, and therapeutic evaluation. All procedures performed in this study involving human participants were in accordance with the Declaration of Helsinki (as revised in 2013). This study is a retrospective analysis of multicenter data, ethical approval and informed consent is waived.

\section{Clinical data collection and study population}

The study flow diagram is presented in Figure 1. This retrospective study enrolled patients with ESCC who were treated with definitive radiotherapy with or without chemotherapy between February 2017 and December 2001. The following inclusion criteria were applied: (I) new diagnosis of ESCC; (II) clinical stage I-IV ESCC according to the $8^{\text {th }}$ edition of the American Joint Committee on Cancer (AJCC) tumour/node/metastasis classification; and (III) treatment with definitive radiotherapy using 3DCRT or IMRT. A total of 2,762 ESCC patients were identified in the initial study population and the following 


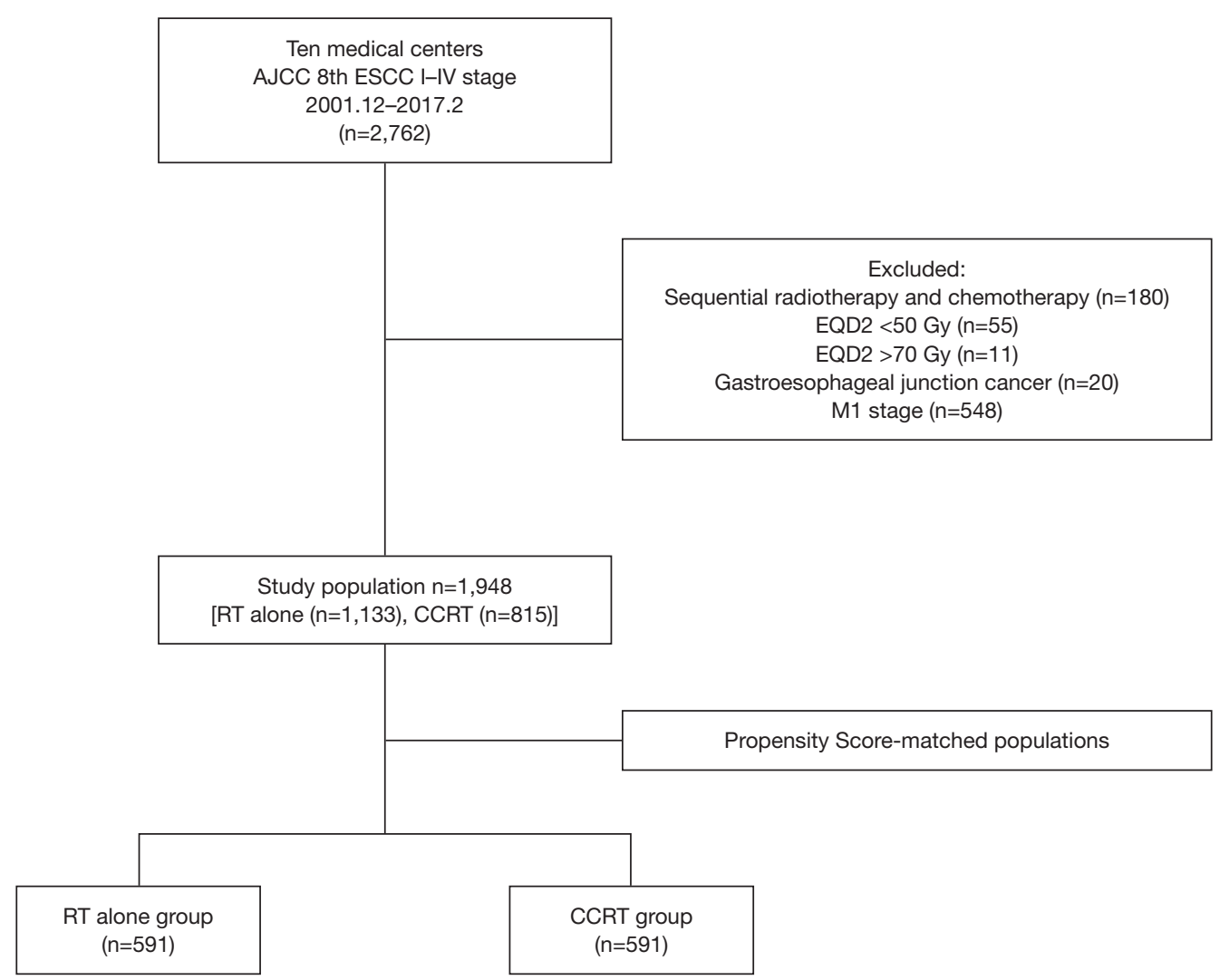

Figure 1 Study flow diagram. AJCC, American Joint Committee on Cancer; ESCC, esophageal squamous cell carcinoma; GTV, gross tumor volume; EQD2, Equivalent Dose in 2 Gy/f; RT, radiotherapy; CCRT, concurrent chemoradiotherapy.

exclusion criteria were applied: (I) treatment with sequential radiotherapy and chemotherapy; (II) radiation dose (EQD2) $<50$ or $>70$ Gy; (III) gastroesophageal junction cancer; (IV) stage M1 cancer; (V) Eastern Cooperative Oncology Group performance status score $>2$; (VI) history of malignant neoplasm; and (VII) severely impaired renal, hepatic, cardiac, or respiratory function.

Following the inclusion and exclusion criteria, a total of 1,948 patients with ESCC who were treated with 3DCRT/IMRT were enrolled in the study. From this, 1,133 patients were categorized into the RT alone group and 815 patients were categorized into the CCRT group. To adjust for imbalances between the two groups, propensity-score matching (PSM) was performed. Standardized differences were calculated for each matched variable (Table 1). Finally, 1,182 patients (591 per treatment group) were matched.

Clinical TNM stage according to $8^{\text {th }}$ edition of the American Joint Committee on Cancer (AJCC) tumor/ node/metastasis (TNM) classification was used in this study, and the c $\mathrm{T}, \mathrm{cN}, \mathrm{cM}$ stages were defined by results obtained from endoscopic ultrasonography (EUS), computed tomography (CT), diffusion weighted magnetic resonance imaging (DWMRI), or positron emission tomography (PET)-CT.

\section{Radiotherapy}

Among the total study cohort of 1,948 patients, 652 and 1,296 patients received 3DCRT and IMRT, respectively. All patients underwent computed tomograph (CT)-based simulation. The scanned images were transferred to a three-dimensional (3D) planning system. Target volume were delineated by physicians using all possible resources (CT, endoscope/EUS, barium esophagram, PET-CT, etc.). Radiotherapy included conventional fractionated radiotherapy and simultaneous integrated boost IMRT. The total radiation dose range of EQD2 was 50-70 Gy. The dose range of the planning target volume (PTV) was 1.6-2.2 Gy per fraction, and $\mathrm{PTV}_{\mathrm{G}_{\mathrm{G}}}$ was 2.0-2.26 Gy. The dose 
Table 1 Characteristics of the study population

\begin{tabular}{|c|c|c|c|c|c|c|}
\hline Characteristic & \multicolumn{3}{|c|}{ Before PSM $(n=1,948)$} & \multicolumn{3}{|c|}{ After PSM $(n=1,182)$} \\
\hline Gender (n) & & & $<0.001$ & & & 0.274 \\
\hline Male & $713(62.9 \%)$ & $616(75.6 \%)$ & & $413(69.9 \%)$ & $430(72.8 \%)$ & \\
\hline Female & $420(37.1 \%)$ & $199(24.4 \%)$ & & $178(30.1 \%)$ & $161(27.2 \%)$ & \\
\hline$<70$ & $509(44.9 \%)$ & $665(81.6 \%)$ & & $434(73.4 \%)$ & $442(74.8 \%)$ & \\
\hline$\geq 70$ & $624(55.1 \%)$ & $150(18.4 \%)$ & & $157(26.6 \%)$ & $149(25.2 \%)$ & \\
\hline Median [range] & $71[37-90]$ & $61[30-88]$ & & 64 [37-90] & 64 [39-88] & \\
\hline \multicolumn{2}{|c|}{ Lesion length (by barium meal, cm) } & & 0.732 & & & 0.639 \\
\hline Median (range) & $5.0(1.0-17.1)$ & $5.0(1.0-18.0)$ & & $5.0(1.0-16.8)$ & $5.0(1.0-18.0)$ & \\
\hline Tumour site $(n)$ & & & $<0.001$ & & & 0.168 \\
\hline Cervical & $46(4.1 \%)$ & $64(7.9 \%)$ & & $27(4.5 \%)$ & $38(6.5 \%)$ & \\
\hline Upper thoracic & $284(25.0 \%)$ & $273(33.5 \%)$ & & $189(32.0 \%)$ & $184(31.1 \%)$ & \\
\hline Middle thoracic & $548(48.4 \%)$ & $324(39.8 \%)$ & & $276(46.7 \%)$ & $250(42.3 \%)$ & \\
\hline Lower thoracic & 255 (22.5\%) & $154(18.9 \%)$ & & $99(16.8 \%)$ & $119(20.1 \%)$ & \\
\hline AJCC T stage (n) & & & 0.029 & & & 0.106 \\
\hline $\mathrm{T}_{1}$ & $18(1.6)$ & $12(1.5)$ & & $9(1.5)$ & $9(1.5)$ & \\
\hline $\mathrm{N}^{+}$ & $687(60.6 \%)$ & $534(65.5 \%)$ & & $373(63.1 \%)$ & 377 (63.8\%) & \\
\hline GTV volume $\left(\mathrm{cm}^{3}\right)$ & & & 0.054 & & & 0.081 \\
\hline$\leq 40$ & 577 (50.9\%) & $379(46.5 \%)$ & & 304 (51.4\%) & $274(46.4 \%)$ & \\
\hline$>40$ & $556(49.1 \%)$ & $436(53.5 \%)$ & & $287(48.6 \%)$ & $317(53.6 \%)$ & \\
\hline Median (range) & $39.6(2.1-293.8)$ & $42.0(3.0-317.2)$ & & $39.6(3.7-293.8)$ & $41.9(3.0-317.2)$ & \\
\hline EQD2 (Gy) & & & 0.906 & & & 0.291 \\
\hline Median & 60 & 60 & & 60 & 60 & \\
\hline Range & $50-70$ & $50-70$ & & $50-70$ & $50-70$ & \\
\hline
\end{tabular}

${ }^{*}, \chi^{2}$ or two-independent-samples tests. PSM, propensity score matching; RT, radiotherapy; CCRT, concurrent chemoradiotherapy; AJCC, American Joint Committee on Cancer; GTV, gross tumor volume; EQD2, Equivalent Dose in 2 Gy/f. 
received by $95 \%$ of the PTV should be more than $100 \%$ of the prescription dose. The organs at risk had the following dose restrictions: lungs, V5 $\leq 55-60 \%$, V20 $\leq 25-30 \%$, V30 $\leq 18 \%$; spinal cord, maximum dose $<45$ Gy; heart, mean dose $\leq 26-30$ Gy.

\section{Chemotherapy}

In this study cohort, 815 patients received chemotherapy concurrently with radiotherapy, for the patients who could get chemotherapy regimen, the drugs were within the recommended range of clinical guidelines. Of these, 395 patients received intravenous platinum-based chemotherapy (cisplatin combined with fluorouracil, paclitaxel, or docetaxel) for 21 or 28 days. The treatments were administered concurrently in the $1^{\text {st }}, 4^{\text {th }}$, or $5^{\text {th }}$ week of radiotherapy in these patients. Additionally, 147 patients received single-drug chemotherapy, including paclitaxel, cisplatin, tegafur, or Xeloda (cisplatin intravenous chemotherapy, tegafur, or Xeloda oral chemotherapy, on a weekly or periodic regimen). The details on drug regimens were not clear for the remaining 273 patients.

\section{Outcome measures}

The primary endpoint was long-term survival, and the secondary endpoints were disease-free survival (DFS) and cause of death. The efficacy of concurrent chemotherapy was evaluated by subgroup analyses and multivariate analyses using the Cox regression model.

\section{Statistical analysis}

All statistical analyses were performed using SPSS version 22.0. Overall survival (OS) was assessed using the KaplanMeier method, and differences between subgroups were assessed using the log-rank test. The survival of patients who were lost to follow-up was calculated as a truncated value according to the last follow-up date. A propensityscore-matched cohort was constructed to balance observable potential confounders and the patients in the radiotherapy alone group were screened and matched with the CCRT group by PSM with a ratio of 1:1. The matching variables included gender, age, lesion length, tumor site, clinical T stage, N stage, TNM stage, gross tumor volume (GTV) volume, and radiation dose (EQD2). The adopted calliper width was 0.02 and $\mathrm{P}<0.05$ was considered statistically significant.

\section{Results}

\section{Baseline characteristics of the patients}

There were differences in the baseline characteristics between the RT alone group and the CCRT group (Table 1). In the CCRT group, there was a higher proportion of males, younger patients, cervical and upper thoracic cancers, and worse $\mathrm{T}$ and $\mathrm{N}$ stages. To adjust for imbalances between the two groups, PSM was performed based on gender, age, lesion length determined by the barium meal, tumor site, AJCC T stage, AJCC N stage, and GTV. Standardized differences were calculated for each matched variable (Table 1). Finally, 1,182 patients (591 per treatment group) were matched.

\section{Therapeutic effects (TEs)}

After PSM, 391 patients in the RT alone cohort had sufficient TE evaluation information, including 144 patients with complete response (CR; 36.8\%), 227 with partial response (PR; 58.1\%), 19 with stable disease (SD; 4.9\%), and 1 with progressive disease (PD; $0.2 \%$ ).

In the CCRT group, 253 patients had sufficient TE evaluation information, including 71 with CR (28.1\%), 158 with PR (62.5\%), 23 with SD (9.1\%), and 1 patient with PD (0.3\%).

The overall response rate (CR + PR) of the RT alone group and the CCRT group was $94.5 \%$ and $90.5 \%$, respectively. The disease control rate $(\mathrm{CR}+\mathrm{PR}+\mathrm{SD})$ of the two groups were $99.7 \%$ and $99.6 \%$, respectively.

\section{Survival analyses}

The Kaplan-Meier analysis revealed that the 1-, 3-, 5-, 7-, and 10 -year OS rates were $73.5 \%, 39.1 \%, 29.1 \%, 21.7 \%$, and $14.9 \%$, respectively, in the radiotherapy alone group and $76.2 \%, 48.1 \%, 39.9 \%, 29.3 \%$, and $27.1 \%$, respectively, in the CCRT group. The median survival times before PSM were 24.1 and 31.2 months, respectively $\chi^{2}=15.451$, $\mathrm{P}<0.001$; Figure $2 A$ ). The OS of the CCRT group before PSM was significantly higher than that of the radiotherapy alone group.

After PSM, the 1-, 3-, 5-, 7-, and 10-year OS rates were $75.4 \%, 50.7 \%, 44.6 \%, 39.4 \%$, and $34.0 \%$, respectively, in the radiotherapy alone group and $77.5 \%, 49.0 \%, 41.9 \%$, 
A

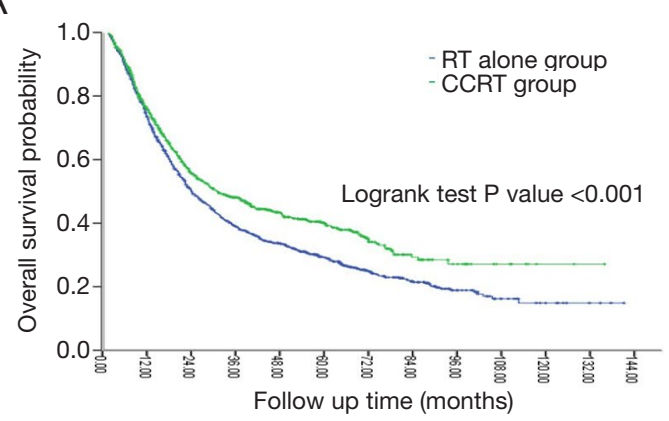

C

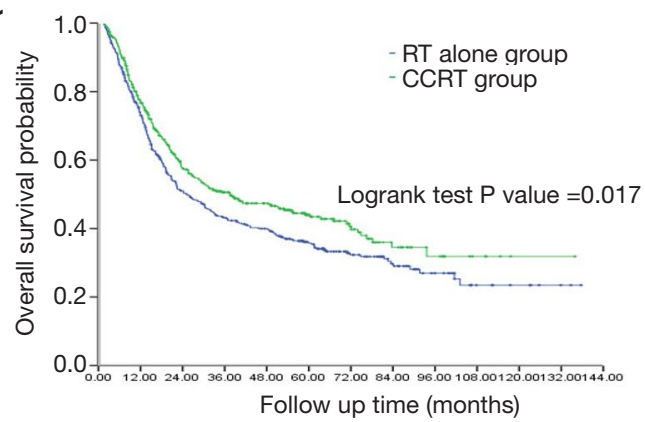

B

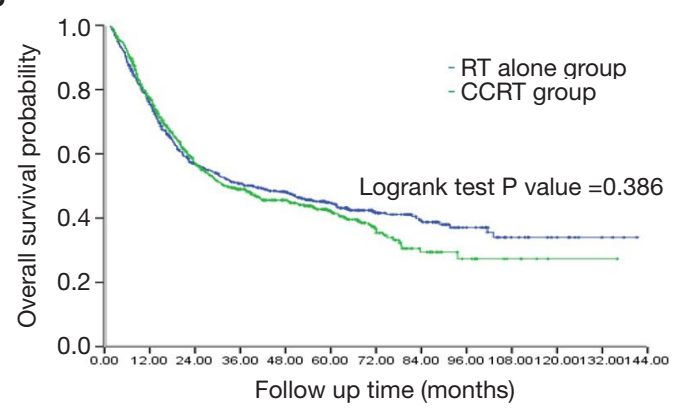

D

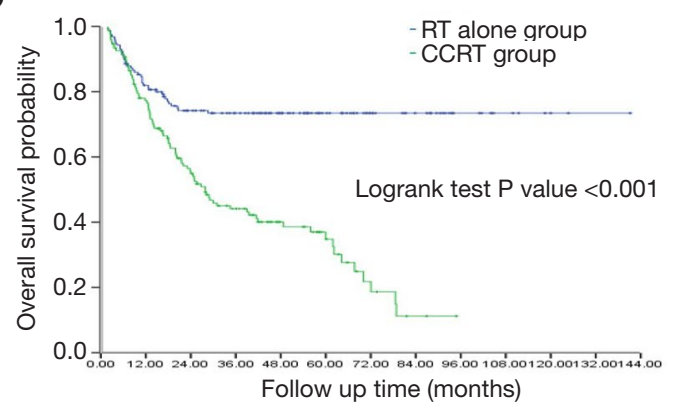

Figure 2 The Kaplan-Meier curves of the patients in the RT alone group and the CCRT groups before propensity-score matching (A) and after propensity-score matching (B). The Kaplan-Meier curves for patients who are younger than 70 years (C) and older than 70 years (D) in the different treatment groups. RT, radiotherapy; CCRT, concurrent chemoradiotherapy.

$29.4 \%$, and $27.3 \%$, respectively, in the CCRT group. The median survival rates were 39.4 and 32.6 months, respectively $\left(\chi^{2}=0.751, \mathrm{P}=0.386\right.$; Figure $\left.2 B\right)$. The 5 - and 10 -year DFS rates were $40.8 \%$ and $28.4 \%$, respectively, in the radiotherapy alone group and $35.3 \%$ and $30.5 \%$, respectively, in the CCRT group. The median progressionfree survival rates were 25.4 and 19.2 months, respectively $\left(\chi^{2}=3.334, \mathrm{P}=0.068\right)$. Compared with the radiotherapy alone group, there was no advantage in OS or DFS in the CCRT group after the PSM.

In the matched cohort of 1,182 patients (519 pairs), 876 patients were younger than 70 years of age, including 434 patients treated with radiotherapy alone and 442 patients treated with CCRT. The comparison of the baseline characteristics of these two subgroups revealed no significant difference in gender, tumor length, tumor site, $\mathrm{N}$ stage, GTV volume, or EQD2, however, there was a significant difference in T stage. PSM was performed again to adjust for the imbalance, with a 1:1 ratio and a calliper width of 0.5 . After the second PSM, there were 434 patients per group. The 1-, 3-, 5-, 7-, and 10-year OS rates were $73.0 \%, 43.1 \%, 35.7 \%, 29.7 \%$ and $23.5 \%$, respectively, in the radiotherapy alone group and $77.4 \%, 50.6 \%, 44.0 \%$,
$34.5 \%$, and $31.9 \%$, respectively, in the CCRT group. The median OS times were 25.1 and 37.5 months, respectively $\left(\chi^{2}=5.683, \mathrm{P}=0.017\right)$. In patients younger than 70 years old, the OS of the CCRT group was significantly higher than that of the radiotherapy alone group. The median OS benefit was approximately 12.4 months (Figure $2 C$ ).

The remaining 306 patients who were 70 years or older included 157 patients in the radiotherapy alone group and 149 patients in the CCRT group. There were no significant differences in the baseline characteristics between these two subgroups. The 1-, 3-, 5-, 7-, and 10-year OS rates were $82.0 \%, 73.4 \%, 73.4 \%, 73.4 \%$, and $73.4 \%$, respectively, in the radiotherapy alone group and $77.3 \%, 44.1 \%, 34.8 \%$, and $11.2 \%$, respectively, in the CCRT group. The median OS of the CCRT group was 27.9 months $\left(\chi^{2}=37.280\right.$, $\mathrm{P}<0.001)$. The 10 -year OS rate of the CCRT group and the median OS time of the RT alone group did not reach. In the patients who were 70 years or older, the OS of the radiotherapy alone group was significantly higher than that of the CCRT group (Figure 2D).

The study examined the effects of the chemotherapy protocol on survival. After PSM, there were 269 patients in the CCRT cohort who received combination chemotherapy 
of two drugs and 115 patients received single-drug chemotherapy. The details on the drug regimens were unclear for the remaining 207 patients. The median survival time (MST) of the two-drug combination chemotherapy group and the single-drug chemotherapy group were 48.5 months and 23.1 months, respectively, with the former performing significantly better than the latter $\left(\chi^{2}=4.604\right.$, $\mathrm{P}=0.032$ ). For patients younger than 70 years old, there was no significant advantage in the combination of two drugs compared with single drug treatment (MST $41.1 \mathrm{vs.}$ 30.1 months, respectively; $\chi^{2}=0.503, \mathrm{P}=0.478$ ).

\section{Multivariate analysis}

The Cox regression analysis to determine the univariate and multivariate prognosis of the matched datasets revealed that the prognostic patterns of patients younger than 70 years and those 70 years or older were different (Tables 2 and 3). The common independent prognostic factors were $\mathrm{T}$ and $\mathrm{N}$ stages, and the prognosis was poorer in patients with advanced $\mathrm{T}$ or $\mathrm{N}$ stage ESCC. In addition, gender and GTV volume were independent prognostic factors in the subgroup of patients younger than 70 years of age. Females were more likely to benefit from treatment [hazard ratio (HR) $0.787,95 \%$ confidence interval (CI) 0.639 to 0.970 , $\mathrm{P}=0.025]$. In addition, a larger GTV volume predicted poorer prognosis (HR 1.452, 95\% CI: 1.181 to 1.784 , $\mathrm{P}<0.001$ ).

It was noteworthy that the intervention with CCRT had different prognostic effects on patients in different age groups. Specifically, in younger patients ( $<70$ years), CCRT provided survival benefit (HR $0.782,95 \%$ CI: 0.657 to $0.932, \mathrm{P}=0.006$ ) and prolonged the median survival time by 12.4 months. However, in elderly patients ( $\geq 70$ years), CCRT was an independent poor prognostic risk factor (HR $3.206,95 \%$ CI: 2.168 to $4.740, \mathrm{P}<0.001)$.

\section{Analysis of the cause of death}

During the study period until the follow-up date, 523 of the 868 patients younger than 70 years of age died, including 284 patients in the radiotherapy alone group and 229 patients in the CCRT groups. Out of the 306 patients who were 70 years of age or older, 131 died during the study period, including 40 patients who were treated with radiotherapy alone and 91 patients who were treated with CCRT. The most common cause of death was local failure including uncontrolled tumor or recurrence. Metastasis was the second most frequent cause of death. The rate of death caused by perforation and bleeding in the radiotherapy alone group was significantly higher than that in the CCRT group (Table 4) for both patients who were younger than 70 years $\left(18.6 \%\right.$ vs. $\left.11.4 \%, \chi^{2}=5.197, \mathrm{P}=0.023\right)$ and those who were 70 years or older $\left(17.5 \%\right.$ vs. $3.3 \%, \chi^{2}=6.063$, $\mathrm{P}=0.014)$.

\section{Discussion}

The treatment regimen of radiotherapy with 50-50.4 Gy combined with concurrent administration of fluorouracil and cisplatin has been recommended in the National Comprehensive Cancer Network (NCCN) guidelines for many years. The evidence is derived mainly from the RTOG8501 $(1,2,3)$ and RTOG9405 (4) trials. However, the TE of radiotherapy alone in the RTOG8501 trial was very poor (5-year survival rate was $0 \%$ ), which was not in line with the clinical experience and historical data. With two-dimensional radiotherapy used extensively in the last century and the recently implemented 3D technologies, the 5 -year survival rate of patients with esophageal cancer treated with radiotherapy alone has ranged between $8 \%$ and $27.7 \%(5-10)$ based on a large number of reported cases. In patients with early esophageal cancer (tumor length $<3 \mathrm{~cm}$ ), the 5-year survival rate with radiotherapy alone could reach $30.1 \%$ (11). Unfortunately, currently in China, where squamous cell carcinoma is the main pathological type, high-level research evidence to confirm or replicate the results of the RTOG8501 and RTOG9405 trials are lacking.

Therefore, this study retrospectively analyzed 2,762 patients with ESCC who were treated by radiotherapy alone or CCRT in ten medical centres in China. The aim was to evaluate the benefits of CCRT compared with radiotherapy alone in the context of precise radiotherapy and to determine the beneficial subgroups. A total of 1948 patients with non-stage IV ESCC who fulfilled both the inclusion and exclusion criteria were included in the final analyses. There were 1,133 patients treated with radiotherapy alone and 815 patients who were treated with CCRT. The Kaplan-Meier analysis determined that CCRT had a survival advantage over radiotherapy alone and that the median OS benefit of CCRT was approximately seven months in the unmatched cohort of patients with stage IIII ESCC. However, there were differences in the baseline characteristics of the two groups, with the CCRT group showing a higher proportion of males, younger patients, 
Table 2 Analysis of the prognostic factors in patients younger than 70 years of age $(n=868)$

\begin{tabular}{|c|c|c|c|c|c|c|c|c|}
\hline Characteristic & Cases & \multicolumn{4}{|c|}{ Univariate analysis } & \multicolumn{3}{|c|}{ Multivariate analysis } \\
\hline \multicolumn{9}{|l|}{ Gender } \\
\hline Male & 625 & 25.1 & 1 & (Reference) & (Reference) & 1 & (Reference) & (Reference) \\
\hline Female & 243 & 55.2 & 0.690 & $0.564-0.844$ & $<0.001$ & 0.787 & $0.639-0.970$ & 0.025 \\
\hline$\leq 5$ & 474 & 40.1 & 1 & (Reference) & (Reference) & 1 & (Reference) & (Reference) \\
\hline$>5$ & 394 & 21.8 & 1.396 & $1.174-1.660$ & $<0.001$ & 1.051 & $0.868-1.272$ & 0.612 \\
\hline \multicolumn{9}{|l|}{ Tumour site } \\
\hline Cervical/upper thoracic & 327 & 49.0 & 1 & (Reference) & (Reference) & 1 & (Reference) & (Reference) \\
\hline $\mathrm{T}_{1+2}$ & 165 & 83.5 & 1 & (Reference) & (Reference) & 1 & (Reference) & (Reference) \\
\hline $\mathrm{T}_{3}$ & 296 & 35.2 & 1.368 & $1.035-1.809$ & 0.028 & 1.137 & $0.855-1.514$ & 0.377 \\
\hline $\mathrm{T}_{4}$ & 407 & 20.7 & 1.985 & $1.530-2.576$ & $<0.001$ & 1.482 & $1.123-1.955$ & 0.005 \\
\hline \multicolumn{9}{|l|}{ AJCC N stage } \\
\hline $\mathrm{N}_{0}$ & 298 & 67.1 & 1 & (Reference) & (Reference) & 1 & (Reference) & (Reference) \\
\hline $\mathrm{N}_{+}$ & 570 & 22.4 & 1.657 & $1.366-2.011$ & $<0.001$ & 1.455 & $1.191-1.777$ & $<0.001$ \\
\hline \multicolumn{9}{|l|}{ GTV volume $\left(\mathrm{cm}^{3}\right)$} \\
\hline$\leq 40$ & 399 & 62.3 & 1 & (Reference) & (Reference) & 1 & (Reference) & (Reference) \\
\hline \multicolumn{9}{|l|}{ Radiotherapy (EQD2) (Gy) } \\
\hline \multicolumn{9}{|l|}{ Treatment regimen } \\
\hline RT alone & 434 & 25.1 & 1 & (Reference) & (Reference) & 1 & (Reference) & (Reference) \\
\hline CCRT & 434 & 37.5 & 0.809 & $0.680-0.963$ & 0.017 & 0.782 & $0.657-0.932$ & 0.006 \\
\hline
\end{tabular}

MST, mean survival time; HR, hazard ratio; Cl, confidence interval; AJCC, American Joint Committee on Cancer; GTV, gross tumor volume; EQD2,Equivalent Dose in 2 Gy/f; RT, radiotherapy; CCRT, concurrent chemoradiotherapy.

cervical and upper thoracic cancers, and worse $\mathrm{T}$ and $\mathrm{N}$ stages, all of which were prognostic survival factors. Therefore, to eliminate the interference of confounding factors and to bring this study closer in line with the quality of prospective studies, PSM with a ratio of $1: 1$ was performed using the baseline characteristics of the two groups. A total of 1,182 matched patients (591 pairs) were obtained. The analyses after the PSM showed that there was no significant difference in the OS between the two groups. The median OS times and the 5-year survival rates were 32.6 and 39.4 months, and $41.9 \%$ and $44.6 \%$ in the CCRT and radiotherapy alone groups, respectively. These findings indicated that CCRT did not provide additional benefits compared to RT alone in the cohort of 
Table 3 Analysis of the prognostic factors in patients older than 70 years of age $(n=306)$

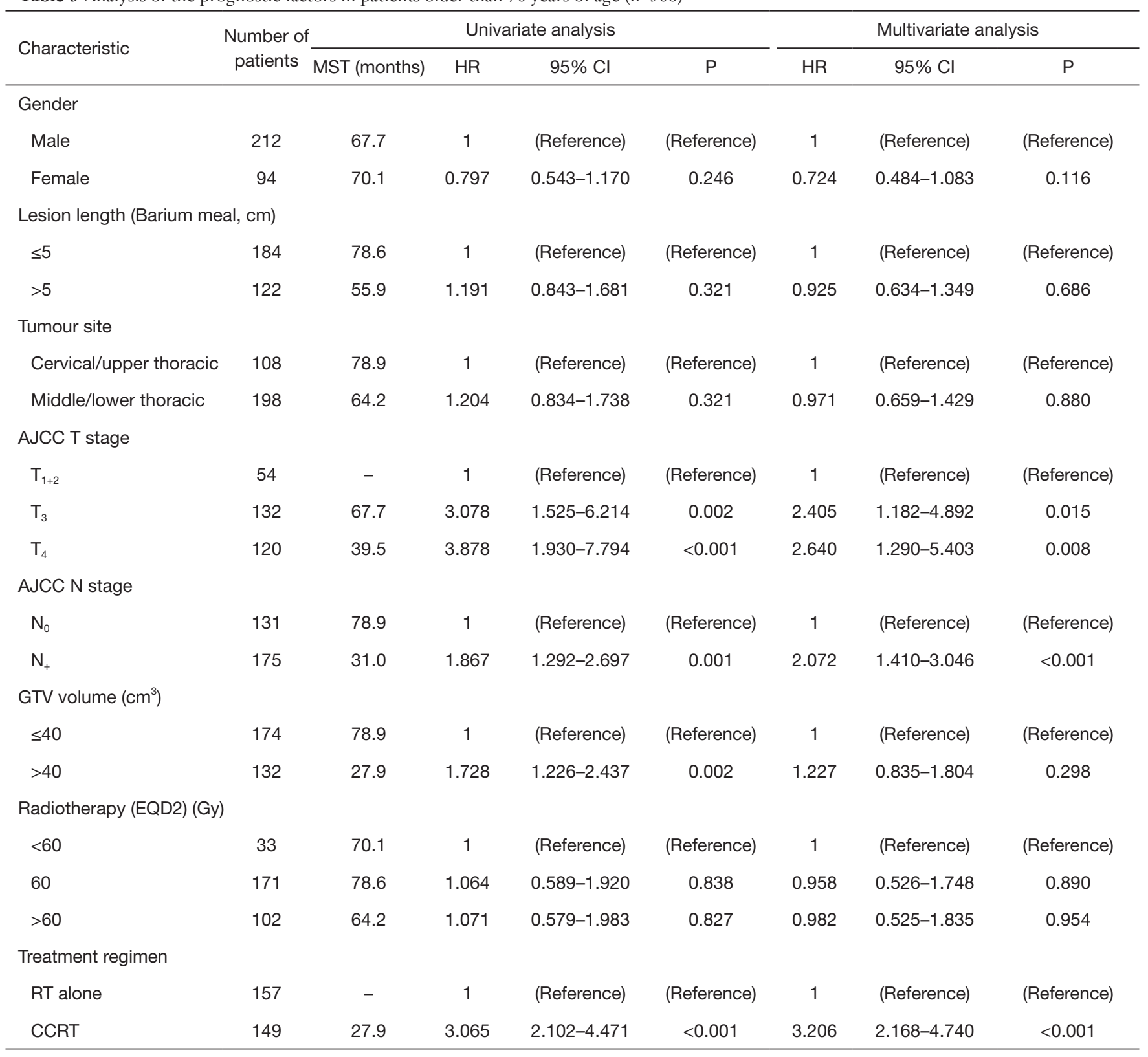

MST, mean survival time; HR, hazard ratio; Cl, confidence interval; AJCC, American Joint Committee on Cancer; GTV, gross tumor volume; EQD2, Equivalent Dose in 2 Gy/f; RT, radiotherapy; CCRT, concurrent chemoradiotherapy.

patients who were matched for the baseline characteristics. Further analyses of the characteristics of the two matched groups revealed that age was a key factor to the survival benefit of CCRT. Specifically, in patients younger than 70 years of age, the median OS times and the 5 -year survival rates were 37.5 vs. 25.1 months, and $44.0 \%$ vs. $35.7 \%$ in the CCRT and the radiotherapy alone groups, respectively. The median OS benefit of CCRT was therefore 12.4 months. In patients aged 70 years or older, contrary results were observed. Specifically, the 5-year survival rates were $34.8 \%$ and $73.4 \%$ in the CCRT and radiotherapy alone groups, respectively, indicating that the OS rate of radiotherapy alone was evidently superior to that of CCRT. Further multivariate analyses showed that 
Table 4 An analysis of the cause of death for patients treated with the different modalities

\begin{tabular}{|c|c|c|c|c|}
\hline Group & \multicolumn{2}{|c|}{ Age $<70$ years $(n=868)$} & \multicolumn{2}{|c|}{ Age $\geq 70$ years $(n=306)$} \\
\hline Local failure & $78(27.5)$ & $70(30.6)$ & $12(30.0)$ & $34(37.3)$ \\
\hline Regional failure & $7(2.5)$ & $20(8.7)$ & $3(7.5)$ & $7(7.7)$ \\
\hline Hemorrhage & 35 (12.3) & $18(7.9)$ & $6(15.0)$ & $1(1.1)$ \\
\hline Perforation & $18(6.3)$ & $8(3.5)$ & $1(2.5)$ & $2(2.2)$ \\
\hline Treatment-related death & 0 & $1(0.4)$ & $1(2.5)$ & $1(1.1)$ \\
\hline
\end{tabular}

*, two or more types of local failure, regional failure, and metastasis. RT, radiotherapy; CCRT, concurrent chemoradiotherapy.

CCRT provided survival benefit in patients younger than 70 years of age and was associated with a $21.8 \%$ reduction in the risk of death. However, in elderly patients ( $\geq 70$ years) CCRT was an independent poor prognostic risk factor (HR $=3.206, \mathrm{P}<0.001)$ and might significantly increase the risk of death. We speculate that the negative efficacy of CCRT in group older than 70 years is related to the high toxicity of the treatment, exceeding the patient's tolerance, and the inhibiting of immunogenomic function. Therefore, CCRT may not be suitable for all patients with ESCC in China, while younger patients may benefit significantly from CCRT.

The present study has several limitations that should be considered in the interpretation of the findings. First, the region included in the study is large, encompassing ten hospitals, and the time span is long, encompassing the period between 2001 and 2017. These factors may lead to differences in radiotherapy technology, equipment, and medication regimens, which may interfere with the comparison of results. In order to reduce the interference of confounding factors, the researchers enhanced the quality control of enrolled cases and screening of inclusion and exclusion criterias, and used propensity score matching method to balance the clinicopathological and treatment factors. Second, chemotherapy regimens were not uniform and included two agents combined or a single agent. The combinations of chemotherapy and radiotherapy also varied greatly, including 28- or 21-day cycles, as well as weekly or 2 -week regimens. In addition, the details of the regimens were not clear for 273 patients. These factors might have affected the efficacy and the observed toxicity of CCRT. Third, in the analysis of the older patient group, the TEs of CCRT were inferior to that of radiotherapy alone. Interestingly, the median OS of CCRT was not considered too short (27.9 months) even though the OS rates were evidently superior in the radiotherapy alone group. We speculate that this may be related to the selection bias of the retrospective analysis. Based on the uncertainties in the above retrospective analysis, it is necessary to carry out prospective studies to determine the safety and survival benefit of concurrent chemoradiotherapy in elderly patients.

Regarding the survival benefit of CCRT in elderly patients, a single-centre retrospective analysis (12) has previously shown that age 70 years or older is a poor prognostic factor $(\mathrm{HR}=2.099, \mathrm{P}=0.001)$ for CCRT, however, all patients in the CCRT group were treated with the cisplatin plus 5-florouracil (FP) or paclitaxel plus cisplatin (TP) regimen in this study, and the results might indicate that two-drug intravenous chemotherapy in combination with concurrent radiotherapy does not provide survival benefit in elderly patients. In contrast, single-drug regimens, especially S-1 as a single oral agent, in combination with concurrent radiotherapy for elderly patients with esophageal cancer has received much focus in recent years (13-15). Therefore, for elderly patients aged 70 years or older, the potential survival benefit of CCRT, as well as optimal treatment regimens require further research 
to guide clinical treatment options.

\section{Acknowledgments}

Funding: None.

\section{Footnote}

Reporting Checklist: The authors have completed the STROBE reporting checklist. Available at https://dx.doi. org/10.21037/tcr-21-505

Data Sharing Statement: Available at https://dx.doi. org/10.21037/tcr-21-505

Conflicts of Interest: All authors have completed the ICMJE uniform disclosure form (available at https://dx.doi. org/10.21037/tcr-21-505). The authors have no conflicts of interest to declare.

Ethical Statement: The authors are accountable for all aspects of the work in ensuring that questions related to the accuracy or integrity of any part of the work are appropriately investigated and resolved. All procedures performed in this study involving human participants were in accordance with the Declaration of Helsinki (as revised in 2013). This study is a retrospective analysis of multicenter data, ethical approval and informed consent is waived.

Open Access Statement: This is an Open Access article distributed in accordance with the Creative Commons Attribution-NonCommercial-NoDerivs 4.0 International License (CC BY-NC-ND 4.0), which permits the noncommercial replication and distribution of the article with the strict proviso that no changes or edits are made and the original work is properly cited (including links to both the formal publication through the relevant DOI and the license). See: https://creativecommons.org/licenses/by-nc-nd/4.0/.

\section{References}

1. Herskovic A, Martz K, al-Sarraf M, et al. Combined chemotherapy and radiotherapy compared with radiotherapy alone in patients with cancer of the esophagus. N Engl J Med 1992;326:1593-8.

2. Al-Sarraf M, Martz K, Herskovic A, et al. Progress report of combined chemoradiotherapy versus radiotherapy alone in patients with esophageal cancer: An intergroup study. J
Clin Oncol 1997;15:277-84.

3. Cooper JS, Guo MD, Herskovic A, et al.

Chemoradiotherrapy of locally advanced esophageal cancer: long-term follow-up of a prospective randomized trial (RTOG 85-01). Radiation Therapy Oncology Group. JAMA 1999;281:1623-7.

4. Minsky BD, Pajak TF, Ginsberg RJ, et al. INT 0123(Radiation Therapy Oncology Group 94-05) phase III trial of combined-modality therapy for esophageal cancer: high-dose versus standard-dose radiation therapy. J Clin Oncol 2002;20:1167-74.

5. Delcambre C, Jacob JH, Dottier D, et al. Localized squamous-cell cancer of the esophageal: Retrospective analysis of three treatment schedules Intensity Modulated Radiotherapy. Radiother Oncol 2001;59:195-201.

6. Zhu XZ, Yin SL, Chen QL. Raiotherapy of Carcinoma of Esophagus-An analysis of 2722 cases. Chin J Radiat Oncol 1988;2:18-9.

7. Yin W, Zhang L, Yang Z, et al. Clinical analysis of 3798 cases of esophageal cancer treated by radiotherapy. Chin J Radiat Oncol 1980;3:216-20.

8. Radiotherapy Department of Shanghai Cancer Hospital: Clinical analysis of 1034 cases of esophageal cancer treated by radiotherapy. Cancer Research on Prevention and Treatment 1978;4:46-51.

9. Wang Lan, Han Chun, Liu Lihong, et al. Clinical features of esophageal cancer patients with long-term survival and related factors for treatment. Chin J Radiat Oncol 2016;25:565-70.

10. Wang $X$, Wang L, Chen J, et al. Prognostic analysis of definitive three-dimensional radiotherapy for nonsurgically resectable esophageal squamous cell carcinoma: a multi-centre retrospective study (3JECROG R-01). Chin J Radiat Oncol 2018;27:959-64.

11. Chen Q, Han C, Zhu X. Analysis of treatment effect for patients with lesions of $3 \mathrm{~cm}$ or shorter than $3 \mathrm{~cm}$ esophagus carcinoma. Chin J Radiat Oncol 1997;6:154-6.

12. Wang L, Zhen S, Han C, et al. Optimal radiation dose for esophageal squamous cell carcinoma. Chin J Radiat Oncol 2017;26:1263-8.

13. Li C, Wang X, Wang X, et al. A multicenter phase III study comparing Simultaneous Integrated Boost(SIB) radiotherapy concurrent and consolidated with S-1 versus SIB alone in elderly patients with esophageal and esophagogastric cancer-the 3 JECROG P-01 study protoclo. BMC Cancer 2019;19:397.

14. Ji Y, Du X, Tian Y, et al. A phase II study of S-1 with concurrent radiotherapy in elderly patients with 
esophageal cancer. Oncotarget 2017;8:83022-9.

15. Song GM, Tian X, Liu XL, et al. Concurrent chemoradiotherapy with $\mathrm{S}-1$ as an alternative therapy for elderly Chinese patients with non-metastatic esophageral

Cite this article as: Wang L, Wang X, Ren X, Han C, Xiao Z, Zhu S, Qiao X, Zhou Z, Shen W, Chen J, Pang Q, Zhang W, Zhao Y, Wang X, Sun X, Ge X, Zhang K, Hu M, Li G, Liu M, Wang Y. Age plays an important role in the decision of definitive concurrent chemoradiotherapy (CCRT) for esophageal squamous cell carcinoma (ESCC): a propensityscore matched analysis of multicenter data (3JECROG R-02A). Transl Cancer Res 2021;10(6):2932-2943. doi: 10.21037/tcr21-505 squamous cancer: Evidence based on a systematic review and meta-analysis. Oncotarget 2017;8:37963-73.

(English Language Editor: J. Teoh) 